\title{
The effect of response constraints on the perception of repeating auditory patterns
}

\author{
STEPHEN HANDEL and JAMES D. PICKENS \\ University of Tennessee, Knoxville, Tennessee 37916
}

\begin{abstract}
Subjects were required to identify repeating auditory patterns beginning with or ending with two specified elements. If free to identify and organize these patterns in any manner, subjects organized so that the longest run of identical elements began the pattern. When response constraints were compatible with the preferred organizations (i.e., when the description began with two identical elements), identification was quite accurate. When the response constraints were incompatible with the preferred organizations (i.e., when the description began or ended with two alternating elements), identification was poorer and subjects often resorted to a derived strategy in describing the pattern.
\end{abstract}

The purpose of the present experiment was to investigate the effects of response constraints on the perception of repeating auditory temporal patterns. The patterns were composed of dichotomous elements differing only in pitch. Since a pattern was repeated continuously, it could be started at any one of the elements and still generate the same sequence (e.g., xxo generates the same sequence as does xox or oxx: $x x 0 x x 0 x x o \ldots)$.

Typically, subjects listen to the sequence and are free to organize and verbally describe the sequence as any one of the possible patterns (i.e., xxoxxoxxo ... could be correctly described as xxo, or xox, or oxx). For patterns composed of different pitches, subjects rarely break runs of identical elements and pattern organization can be best conceptualized by considering a pattern as being constructed of two interlocked one-element patterns (xxoxooxo is constructed of $\mathrm{x} x-\mathrm{x}--\mathrm{x}-+--0-00-0)$. One element becomes the "figure" element and subjects then organize the entire pattern around that element according to one of two principles: (1) the run principle, which states that the longest run of identical elements begins the pattern, xxoxooxo or ooxoxxox; or (2) the gap principle, which states that subjects tend to end the pattern with the longest run of the ground element, xoxxoxoo or oxooxoxx (Garner, 1974; Handel, 1974). The preferred organization is a function of presentation rate: At slow rates (2 elements/sec or less) run organization is preferred; at fast rates (4 elements/sec or more) gap organization is preferred (Preusser, 1972).

On this basis, response constraints were chosen which would be compatible and incompatible with unconstrained organizations. For each response constraint, subjects were required to start or end the pattern de-

This research was supported by a National Institutes of Health biomedical support grant to the University of Tennessee. Requests for reprints should be sent to Stephen Handel, Department of Psychology, University of Tennessee, Knoxville, Tennessee 37916. scription with two specified elements. Two types of compatibility can be identified. The first type was based on preferences for a figure element. Given that subjects prefer one of the elements to be the figure, then response constraints that require the figure element to begin the pattern or require the ground element to end the pattern would be compatible, while constraints that require the ground element to begin the pattern or the figure element to end the pattern would be incompatible. The second type of constraint was based on the preference to maintain the integrity of runs of identical elements and could be termed a structural constraint. Here, compatible constraints would require subjects to begin or end the pattern with runs of identical elements, while incompatible constraints would require subjects to begin or end the pattern with an alternation between elements.

\section{METHOD}

\section{Subjects}

The subjects were 12 students attending the University of Tennessee.

\section{Stimulus Presentation}

The patterns were composed of two elements: $595 \mathrm{~Hz}$ for the low element (L) and $795 \mathrm{~Hz}$ for the high element (H). All elements were of equal intensity $(65 \mathrm{~dB})$, of equal duration, and temporal spacing. The patterns were tape recorded and presented monaurally.

The patterns were presented at the rate of 3 elements/sec. This rate was chosen because it was thought to bring about both run and gap organizations (Preusser, 1972). Each element was presented for one-half of the interelement interval.

\section{Patterns}

There were a total of nine patterns. Three patterns were 8 elements long: HHHLLHHL, HHHHLLHL, and HHLLHLHL; three were 9 elements long: HHLHHLLHL, HHHLLHLLL, HHHHHHLLL; and three were 10 elements long: HHHLHLLHHL, HHHHLHLLHL, HHHHHLHLLL. Each pattern and its complement (obtained by exchanging the elements-the complement of HHHHLLHL would be LLLLHHLH) were presented equally often. 


\section{Constraints}

There were eight different response constraints, each requiring subjects to begin or end the pattern with two specified elements. Four constraints required subjects to begin the pattern description with two elements $\mathrm{CHH}$ LL , HL

$\mathrm{LH}_{\text {__ _ }}$ and four constraints required subjects to end the pattern with two elements (__ HH, _ L _ _ _ HL,

LH). The underline schematically represents the remainder of the pattern: If the underline follows the constraint, the constraint must begin the description; if the underline precedes the constraint, the constraint must end the pattern In addition, there was one response condition in which there were no constraints; subjects could describe the pattern in any way they wished.

\section{Experimental Design}

There were a total of 162 conditions: 9 patterns by 2 complements by 9 constraints. These conditions were broken into two sets of 81 by pairing each of the constraints with either the pattern or its complement such that the number of high and low elements was roughly equal. Each subject was presented one of the two sets.

Presentation of the 81 conditions in each set was spread across three sessions. For each session, 27 conditions were presented such that each pattern occurred three times and each constraint occurred three times. Within sessions, the order of conditions was counterbalanced and, similarly, the order of the sessions was counterbalanced.

Before the initiation of each trial, the subject was given a small index card with the constraint written on it. The pattern was presented for $60 \mathrm{sec}$. At this point, the subject verbally described the pattern by stating the sequence of high and low elements. Two performance measures were obtained: (1) the percentage of correct descriptions of the element sequence and, (2) for correct descriptions, the manner in which the element sequence was organized in to a repeating unit.

\section{RESULTS}

Preliminary analyses indicated performance for a pattern and its complement was identical. Moreover, although pattern identification improved markedly across the three sessions $(48 \%, 62 \%$, and $73 \%$ correct for Sessions 1,2, and 3, respectively), the effect of practice did not interact with the experimental variables. On this basis, a pattern and its complement were treated as equivalent and the results were averaged across sessions. The percentage correct for each pattern by constraint condition is shown in Table 1 .

\section{Pattern Identification}

The percentages correct were analyzed in a repeated measures three-way analysis of variance. The only significant terms were the main effects of constraint $[F(8,28)=2.5, p<.05]$ and pattern $[F(8,88)=27.0$, $\mathrm{p}<.01]$. Response constraints which began or ended the pattern with two identical elements were about equal in difficulty to unconstrained responding, and all were easier than constraints which began or ended with two alternating elements $[65 \%$ to $57 \%, \mathrm{t}(6)=3.3$, $\mathrm{p}<.01]$. However, these differences were not large and accounted for only $1 \%$ of the sums of squares.

Pattern difficulty was a function of both pattern length and structural complexity. Although 8-element patterns were easier than 9- or 10-element patterns ( $73 \%$ to $63 \%$ to $48 \%$ ), the difficulties overlapped and in fact the differences were nonsignificant $[F(2,6)=1.1]$. Overall, differences among the nine patterns accounted for $15 \%$ of the sums of squares.

\section{Pattern Organization}

For free responding, individual subjects did not display a preference for beginning the pattern description with one element. Overall, 58\% of the descriptions began with the low pitch tone, $42 \%$ with the high pitch tone. However, there were consistent preferences for run organization. The percentage of run organizations was $58 \%$, the percentage of gap organizations was $26 \%$, and the percentage of organizations which were simultaneously run and gap (e.g., HHHHHLHLLL) was $24 \%$.

The lack of element preferences suggested that the analysis of pattern organization be based on the four

Table 1

Percentage of Correct Identifications for Every Constraint by Pattern Condition

\begin{tabular}{|c|c|c|c|c|c|c|c|c|c|c|}
\hline Pattern & None & $\mathrm{HH}_{-}$ & $\mathrm{LL}_{-}$ & _HH & LL & $\mathrm{HL}_{-}$ & $\mathrm{LH}_{-}$ & HL & LH & $\overline{\mathbf{X}}$ \\
\hline & \multicolumn{10}{|c|}{ 8-Element Patterns } \\
\hline $\begin{array}{l}\text { xxxooxxo } \\
\text { XXXxooxo } \\
\text { Xxooxoxo }\end{array}$ & $\begin{array}{l}58 \\
75 \\
92\end{array}$ & $\begin{array}{l}50 \\
75 \\
83\end{array}$ & $\begin{array}{l}92 \\
75 \\
83\end{array}$ & $\begin{array}{r}58 \\
83 \\
100\end{array}$ & $\begin{array}{l}67 \\
83 \\
83\end{array}$ & $\begin{array}{l}58 \\
67 \\
83\end{array}$ & $\begin{array}{l}58 \\
83 \\
67\end{array}$ & $\begin{array}{l}50 \\
83 \\
58\end{array}$ & $\begin{array}{l}42 \\
75 \\
83\end{array}$ & $\begin{array}{l}59 \\
78 \\
81\end{array}$ \\
\hline & \multicolumn{10}{|c|}{ 9-Element Patterns } \\
\hline $\begin{array}{l}\text { XXoxxooxo } \\
\text { XXX00X000 } \\
\text { XXXXXX000 }\end{array}$ & $\begin{array}{r}58 \\
58 \\
100\end{array}$ & $\begin{array}{r}33 \\
67 \\
100\end{array}$ & $\begin{array}{l}25 \\
67 \\
83\end{array}$ & $\begin{array}{l}33 \\
67 \\
92\end{array}$ & $\begin{array}{l}25 \\
83 \\
92\end{array}$ & $\begin{array}{l}33 \\
50 \\
92\end{array}$ & $\begin{array}{r}42 \\
50 \\
100\end{array}$ & $\begin{array}{l}50 \\
42 \\
75\end{array}$ & $\begin{array}{r}50 \\
42 \\
100\end{array}$ & $\begin{array}{l}39 \\
58 \\
93\end{array}$ \\
\hline & \multicolumn{10}{|c|}{ 10-Element Patterns } \\
\hline 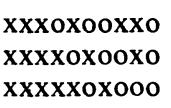 & $\begin{array}{l}42 \\
58 \\
58\end{array}$ & $\begin{array}{l}33 \\
50 \\
58\end{array}$ & $\begin{array}{l}42 \\
50 \\
75\end{array}$ & $\begin{array}{l}42 \\
58 \\
58\end{array}$ & $\begin{array}{l}50 \\
50 \\
75\end{array}$ & $\begin{array}{l}25 \\
33 \\
50\end{array}$ & $\begin{array}{l}25 \\
75 \\
67\end{array}$ & $\begin{array}{l}17 \\
17 \\
75\end{array}$ & $\begin{array}{l}17 \\
50 \\
67\end{array}$ & $\begin{array}{l}32 \\
48 \\
65\end{array}$ \\
\hline$\overline{\mathbf{X}}$ & 67 & 61 & 66 & 66 & 68 & 55 & 62 & 52 & 58 & 62 \\
\hline
\end{tabular}

Note-The percentages of correct identifications are averaged across patterns and their complements. The $x-o$ notation is meant to represent both the pattern and complement. 
structural constraints. The response constraints beginning with two identical elements $\mathrm{HH}_{\mathrm{H}}$ or LL___ should be compatible, as preferred organizations also start with a run of identical elements. Indeed, the pattern descriptions usually began with the longest run $(52 \%)$ or the second longest run $(47 \%)$. The constraints ending with two identical elements (

$\mathrm{HH}$

or _LL) represent an intermediate degree of compatibility-compatible in that it matches preferences not to break up runs of identical elements, yet incompatible as subjects prefer to organize beginning with a run of identical elements. This ambiguity is reflected in performance. Although subjects can usually end the pattern with a pair of identical elements without breaking runs of identical elements, they often must resort to a subterfuge to achieve the constraint. This subterfuge consists of organizing the pattern in the preferred manner, beginning with the run of identical elements and, when verbally responding, placing the first two elements at the end of the pattern. For example, for the constraint $\mathrm{HH}$, the pattern HHHHHLHLLL (the preferred free response) could be described as LHLLLHHHHH, satisfying the constraint and not breaking the run of identical elements. However, $40 \%$ of the responses were HHHLHLLLHH, suggesting the pattern was heard as HHHHHLHLLL and then transformed. Across patterns, $29 \%$ of the organizations were this derived type. Anecdotally, it often looks as though subjects silently mouth the initial elements and then verbalize those elements at the pattern end.

Constraints beginning or ending with an element alternation $\left(\mathrm{HL} \_, \mathrm{LH}_{\longrightarrow}, \quad \mathrm{HL}\right.$, LH) are structurally incompatible. Most of the pattern descriptions did not break runs of identical elements (73\%). However, these constraints may also induce a derived strategy in which subjects use the preferred run organization to get to the required organization. For alternation constraints beginning the pattern description, subjects can "peel" off all but the last element of the initial run and place the beginning elements at the end of the pattern (for the constraint
HL HHHHHLHLLL would be transformed into HLHLLLHHHH). Similarly, for alternation constraints ending the pattern descriptions, subjects can merely move the first element of the run to the end of the pattern (for the constraint LH, HHHHHLHLLL would be transformed into HHHHLHLLLH). Across patterns, these derived organizations (breaking runs of identical elements) account for $27 \%$ of the descriptions.

\section{DISCUSSION}

The two performance measures partition the response constraints in different ways. The identification measure partitions the constraints on the basis of whether the constraints consist of two identical elements or consist of an alternation between elements (higher vs lower percent correct). The pattern description measure partitions the constraints beginning with two identical elements from the other constraints (no-derived vs derived organizations). The results illustrate the relative independence of the two aspects of pattern identification.

The run of identical elements is the basic unit. If the pattern cannot be organized directly by the run, it still serves as the pivotal unit, being transformed to generate the required organization (also see Handel \& Yoder, 1975). However, this does not necessarily produce poorer performance, as the constraint ending with two identical elements was relatively easy. It does suggest that organizational demands can directly influence the difficulty of identification.

\section{REFERENCES}

GARNER, W. R. The processing of information and structure. Potomac, Md: Lawrence Erlbaum, 1974.

Handel, S. Perceiving melodic and rhythmic auditory patterns. Journal of Experimental Psychology, 1974, 103, 922-933.

HANDEl, S., \& Yoder, D. The effects of intensity and interval rhythms on the perception of auditory and visual temporal patterns. Quarterly Journal of Experimental Psychology, 1975, 27, 115-127.

Preusser, D. The effect of structure and rate on the recognition and description of auditory temporal patterns. Perception \& Psychophysics, 1972, 11, 233-240.

(Received for publication November 11, 1976.) 